\title{
ANATOMY OF A PERFORATOR FLAP BASED ON THE SUPERFICIAL CIRCUMFLEX ILIAC ARTERY. A CADAVERIC STUDY
}

\author{
Ahmed Kosba*, Mohamed Mohamed Fata*, Gamal Eldin Borhamy*, \\ Peter Kessler** and Ahmed Serag ElDin Habib*
}

\begin{abstract}
Introduction: The groin flap, based on the superficial circumflex iliac artery (SCIA), was the first successful free flap. It has mainly been used in lower-limb and hand reconstruction with few reports in the literature of its use in soft-tissue head and neck reconstruction.
\end{abstract}

Methods: Five fresh cadaveric dissections were performed and the following parameters were recorded; the presence or absence of the SCIA, and if present, its branching pattern, the diameter of the SCIA and the length of the pedicle.

Results: The superficial circumflex iliac vessels were absent in $40 \%$. When present, the SCIA branches off separately from the femoral artery. A deep branch of the SCIA was found in $40 \%$. The mean diameter at origin was $1.5 \mathrm{~mm}$ on average.

Conclusion: The small vessel calibers and the short pedicle length along with the variable anatomy of the region make the SCIP flap a challenging reconstructive option.

KEY WORDS: Superficial circumflex iliac artery perforator flap; SCIP; head and neck cancer; free flap.

\section{INTRODUCTION}

Defects in the head and neck region can be caused by a number of mechanisms including infection, congenital defects and trauma but the most common cause is following resection for neoplastic disease. ${ }^{(1)}$ Surgical intervention remains the primary treatment modality for the treatment of such neoplasms, but it often results in soft tissue defects necessitating reliable reconstructive procedures..$^{(2)}$
The goals of reconstruction have progressed from filling a hole to providing improved function and appearance and also with the least donor-site morbidity. Free tissue transfer is currently the first choice for reconstruction of large or composite defects in head and neck reconstruction.

The groin flap, supplied by the superficial circumflex iliac artery (SCIA), is one of the first free

* Department of Maxillofacial and Plastic Surgery, Faculty of Dentistry, Alexandria University, Egypt.

** Department of Cranio-Maxillofacial Surgery, Maastricht University Medical Center, The Netherlands 
flaps to be successful in reconstruction. This flap was first described as a pedicled flap by McGregor and Jackson ${ }^{(3)}$ and then introduced as a free flap by Daniel and Taylor. ${ }^{(4)}$ It was the workhorse flap during the early period of microsurgery as the hidden scar and availability of large cutaneous tissue allowed sufficient coverage and the ease of elevation.

The introduction of the perforator flap concept and the evolution of groin flap to superficial circumflex iliac artery perforator (SCIP) flap allowed overcoming some disadvantages such as bulkiness and variable arterial anatomy by using the free style free flap approach.

However, the SCIA is subject to variability as regards the presence, length and caliber, therefore anatomical studies are needed to meticulously study the anatomy of the region before any clinical trials. In this study, we describe our experience with dissection of five fresh frozen cadavers.

\section{METHODS}

In this work we aimed to study the SCIA system and its anatomical variations. Cadavers with scars in the groin region or previously dissected ones were excluded from the study. The anterosuperior iliac spine and the pubic symphysis were used as landmarks. Dissection was initiated medially to easily identify the origin of the SCIA and then continued laterally to identify the superficial and deep branches of the artery, if present. The following parameters were recorded; the presence or absence of the SCIA, and if present, its branching pattern, the diameter of the SCIA and the length of the pedicle.

\section{RESULTS}

In the period between 2016 and 2017, a total of five cadavers had been included in the study.

We found that the superficial circumflex iliac vessels were absent in $40 \%$. In the other $60 \%$, we identified the superficial circumflex iliac vessels where the SCIA branches off the lateral side of the femoral artery, about $1.6-2.5 \mathrm{~cm}$ below the inguinal ligament. It then perforates the deep fascia at a point medial to the lateral border of the Sartorius muscle and courses laterally straight to the anterior superior iliac spine. Its course was found nearly parallel to the inguinal ligament. In 2 out of 3 cadavers, in its course towards the anterior superior iliac spine (ASIS), the SCIA divides into a superficial and a deep branch, of approximately equal size, at $4 \mathrm{~cm}$ from its origin (figure 1). The superficial branch runs parallel to the inguinal ligament and pierces the fascia in a point located at $1.5 \mathrm{~cm}$ medial to the lateral border of the sartorius muscle. Then, it surpasses the ASIS and gives off branches to the skin of the highest portion of the lateral aspect of the thigh.

The mean length of the pedicle that could be dissected was $3.2 \pm 0.8 \mathrm{~cm}$. The mean diameter at origin was $1.5 \pm 0.7 \mathrm{~mm}$. The vena comitant was always smaller with a mean diameter of $0.9 \pm 0.3$ mm (figure 2).

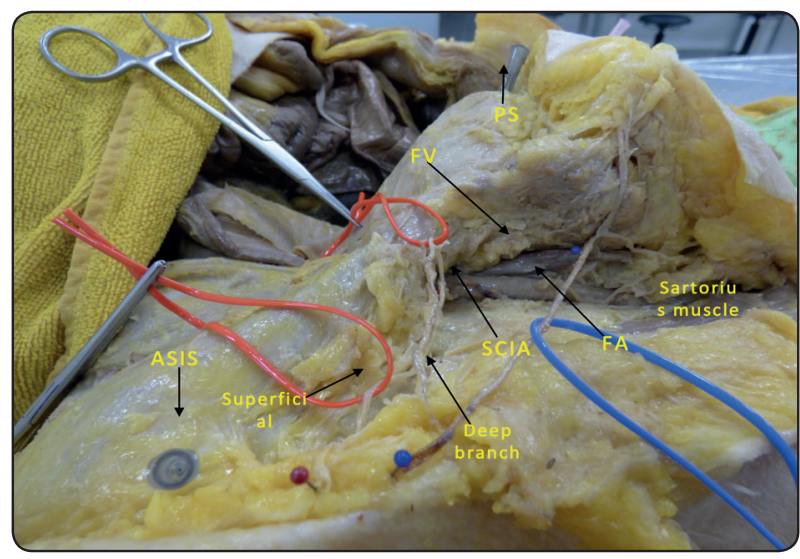

Fig. (1) An anatomical overview of the groin region (right side). ASIS: anterior superior iliac spine, PS: pubis symphysis, FA: femoral artery, FV: femoral vein, SCIA: superficial circumflex iliac artery. 


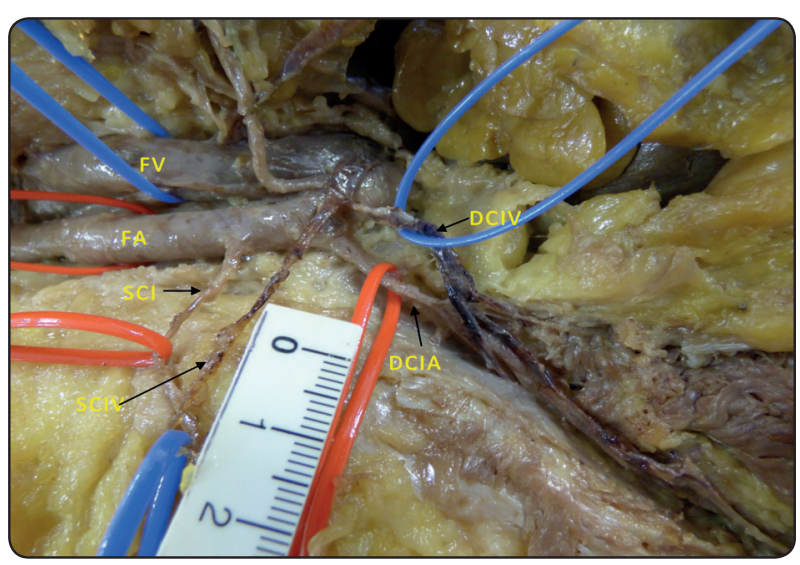

Fig. (2) Vascular anatomy of the groin region (left side). FA: femoral artery, FV: femoral vein, SCIA: superficial circumflex iliac artery, SCIV: superficial circumflex iliac artery, DCIA: deep circumflex iliac artery, DCIV: deep circumflex iliac artery.

\section{DISCUSSION}

The groin flap is of historical importance, however, its popularity was lost due to its variable arterial anatomy. Clinical applications of perforator flap based on SCIA suggest that a dominant branch is enough to supply a groin flap. ${ }^{(5)}$ The development of perforator flap techniques has reduced the donor site morbidity observed with conventional flaps. ${ }^{(6)}$

In 3 dissections of the SCIA, it was found to branch off separately from the femoral artery and then pierces the deep fascia over the sartorius muscle. Therefore, for its clinical application, we suggest that dissection should be started laterally in a retrograde fashion until reaching the sartorius perforator. $^{(7)}$ We also suggest that preoperative imaging of the region (computed tomographic angiography) is necessary to confirm the presence of the SCIA and its perforator. ${ }^{(8)}$ After identification of the perforator, deepithelialization or microsurgical defatting could be done in order to get a thin pliable flap to be transferred to reconstruct orofacial defects. $^{(9)}$
The small vessel caliber of the SCIA necessitates the use of supermicrosurgery techniques to be able to avoid injury of the pedicle while raising the flap and allows for a competent anastomosis. ${ }^{(10)}$

\section{CONCLUSION}

The present anatomical study confirms that the SCIA has an unconstant anatomy. The small vessel calibers and the short pedicle length along with the variable anatomy of the region make the SCIP flap a challenging reconstructive option. Further studies are needed before considering the SCIP flap as a reliable flap to be used in reconstruction of orofacial defects.

\section{REFERENCES}

1. Nasir S, Aydin MA. Versatility of free SCIA/SIEA flaps in the head and neck defects. Ann Plast Surg 2010;65(1): $32-7$.

2. Belcher R, Hayes K, Fedewa S, Chen AY. Current treatment of head and neck squamous cell cancer. J Surg Oncol. 2014;110: 551-574.

3. McGregor IA, Jackson IT. The groin flap. Br J Plast Surg. 1972;25:3-16.

4. Daniel RK, Taylor GI. Distant transfer of an island flap by microvascular anastomoses. A clinical technique. Plast Reconstr Surg 1973;52:111-7.

5. Koshima I, Nanba Y, Tsutsui T, Takahashi Y, Urushibara K, Inagawa K, Hamasaki T, Moriguchi T. Superficial circumflex iliac artery perforator flap for reconstruction of limb defects. Plast Reconstr Surg 2004;113:233-40.

6. Angrigiani C, Grilli D, Siebert J. Latissimus dorsi musculocutaneous flap without muscle. Plast Reconstr Surg 1995;96:1608-14.

7. Green R, Rahman KM, Owen S, Paleri V, Adams J, Ahmed OA, Ragbir M. The superficial circumflex iliac artery perforator flap in intra-oral reconstruction. J Plast Reconstr Aesthet Surg 2013;66:1683-7.

8. He Y, Tian Z, Ma C, Zhang C. Superficial circumflex iliac artery perforator flap: identification of the perforator by computed tomography angiography and reconstruction 
of a complex lower lip defect. Int J Oral Maxillofac Surg. 2015;44(4):419-23.

9. Kimura N, Saitoh M, Hasumi T, Sumiya N, Itoh Y. Clinical application and refinement of the microdissected thin groin flap transfer operation. J Plast Reconstr Aesthet Surg.
2009;62(11):1510-6.

10. Strobbe S, Van Landuyt K, Delaere P, Vander Poorten V, Vanclooster C. Superficial circumflex iliac artery perforator flap for reconstruction of oral defects after tumor resection. B-ENT. 2015;11:157-61. 